\title{
A retrospective cohort study on clinical characteristics of heart failure patients with HFrEF, HFmrEFand HFpEF in geriatrics of Northeastern China
}

\section{Ziwei Zhao}

First Hospital of China Medical University

Cuizhu Hu ( $\sim$ bamboo_huhu@163.com )

First Hospital of China Medical University

\section{Xiaoou Wang}

First Hospital of China Medical University

Wen Tian

First Hospital of China Medical University

\section{Research Article}

Keywords: HFmrEF (Heart failure with mid-range ejection fraction),HFpEF (Heart failure with preserved ejection fraction),HFrEF (Heart failure with reduced ejection fraction), LVEF, China

Posted Date: March 10th, 2021

DOI: https://doi.org/10.21203/rs.3.rs-258985/v1

License: (c) (1) This work is licensed under a Creative Commons Attribution 4.0 International License.

Read Full License 


\section{Abstract}

Background: Heart failure (HF) has been considered as one of the leading cardiovascular disease with high morbidity and high mortality in the world. However, there is a lack of analysis on the clinical characteristics of different types of elderly HF patients in geriatrics of Northeastern China.We conducted this single-center, retrospective study to evaluate the clinical characteristics of HF patients with midrange(HFmrEF), preserved (HFpEF) and reduced ejection fraction (HFrEF) in a Chinese cohort.

Methods: The study was conducted on 1230 patients of age 60 or above, with HF from Jan. 02, 2014 to May 03, 2018 hospitalized in geriatrics department, the First Affiliated Hospital of China Medical University. All the patients met the definition for HFrEF, HFmrEF and HFpEF according to 2016 European Society of Cardiology (ESC) guidelines. Targeted variables including demographics, vital signs, comorbidities and lab assessment results were compared among the three groups by Pearson's $\chi 2$ test, and multivariate logistic regression analyses were performed for risk factor exploration on the three types of HF.

Results: Of the 1230 patients included (78.28 \pm 9.0 years, $67.15 \%$ male), there are 93 patients with $\operatorname{HFrEF}(7.5 \%), 56$ patients with HFmrEF (4.5\%) and 1081 patients with HFpEF (87.8\%). Compared to patients with HFpEF, those with HFmrEF were more likely to have comorbid acute myocardial infarction, infection and coronary artery disease, had higher level of N-terminal pro B-type natriuretic peptide (NTproBNP), cardiac troponin, blood urea nitrogen (BUN), white blood cell (WBC). And patients with HFpEF were more frequently to be female, to be older, and to have comorbid peripheral artery disease, but less frequently had AMI, CAD and kidney disease than those with HFrEF. However, patients with HFmrEF had no significant difference from those with HFrEF. Multivariable analysis showed that comorbid atrial fibrillation,AMI and infection were associated with HFmrEF $(p<0.05)$, whereas female, old age, comorbid hypertension, and PAD were associatedwithHFpEF, and CAD,kidney disease were associated with HFrEF $(p<0.05)$.

Conclusions:The results of our study supported that patients with HFmrEF were closer to patients with HFrEF in respect to most of the demographics andclinical characteristics observed, while showing certain differences from patients with HFpEF.

Trial registration: The study was approved by the local ethics committee from the First Affiliated Hospital of China Medical University(\#2020232,July 13,2020).

\section{Introduction}

Heart failure (HF) is a complex clinical syndrome caused by any structural or functional impairment of ventricular filling orejection of blood[1].It has been considered as one of the major public health concerns with high mortality and economic burden of the patients all over the world. The number of patients hospitalized for HF increases by almost 10,000 per year due to the growing elderly population, and the economic burden has concomitantly increased a lot, correspondingly both in developed and developing 
countries. On the basis of 2016 European Society of Cardiology (ESC) guidelines and left ventricular ejection fraction (LVEF), HF could be classified into 3 types:HFpEF (with preserved EF, LVEF $\geq 50 \%$ ), HFrEF (with reduced $\mathrm{EF}, \mathrm{LVEF}<40 \%$ ) and HFmrEF (with mid-range EF, LVEF of 40-49\%)[3]. Those three categories of HF have different underlying etiologies, demographics, comorbidities and responses to therapies[4]. Approximately $1-2 \%$ of the adultswere diagnosed as HF patientsin developed countries[5]. Especially, a recent study indicated the overall prevalence of HF was $2.2 \%$ in the US, varied from $0.7 \%$ of people aged 45- 54 to $8.4 \%$ of those aged 75 and above [6]. With the aging of population, China will become the country with the most severe aging of population in the world.However,the incidence and epidemiological characteristics of different types of HF are rarely analyzed among the growing elderly population in China. Therefore, we conducted this single-center, retrospective study on patients with these three types of HF in geriatrics departmentof a cohort in Northeastern China.

Our study aims to evaluate the baseline characteristics, comorbidities and laboratory findings, and explore the distribution of HF, and the key risk factors related to those three types of HF in the elderly patients of Northeastern China.

\section{Methods}

\subsection{Study design and population}

Baseline clinical characteristics including age, sex, status of smoking and alcohol use, vital signs including body mass index (BMI), blood pressure and pulse, comorbidities and laboratory findings were compared and analyzed among the three groups of HF patients. BMI was calculated based on the formula $\mathrm{BMI}=$ mass/height. Dyslipidemia was determined by an elevation of the total cholesterol, the lowdensity lipoprotein (LDL) cholesterol and the triglyceride concentrations, and a decrease in the highdensity lipoprotein (HDL) cholesterol in the blood. Coronary artery disease (CAD)consisted of unstable angina pectoris (UAP), comorbid kidney disease (CKD), acute myocardial infarction (AMI) and the other heart diseases.Infection here included all infections throughout the body.

The study was approved by the local ethics committee from the First Affiliated Hospital of China Medical University(\#2020232). All methods were performed in accordance with the relevant guidelines and regulations.

\subsection{Statistical analysis}

Statistical analysis was performed using SAS Statistical Software, Version 9.4 (SAS Institute, Inc., Cary, North Carolina). Continuous variables were presented as mean (SD) and independent t-test was used in comparisons with significance level of 0.05.Categorical variables were calculated as Count (\%) and compared using $\chi^{2}$ test with significance level of 0.05 .Univariate and multivariate logistic regression analyses were used to calculate odds ratio (OR) and $95 \%$ confidence interval, and to find associated factors for the three HF groups. The $p<0.05$ was considered statistically significant. 


\section{Results}

\subsection{Baseline characteristics}

Overall, $1230 \mathrm{HF}$ patients that met the inclusion criteria were identified, of whom 93 patients withHFrEF(7.5\%), 56 patients withHFmrEF (4.5\%) and1081 patients withHFpEF (87.8\%). Baseline characteristics of the total cohort three HF categorieswere presented in Table 1.

The overall population was $78.28 \pm 9.00$ years of age. The difference of age in patients withHFrEF (75.41 \pm 9.23$)$ and those with HFpEF $(78.60 \pm 8.91)$ werestatistically significant $(p<0.05)$. With respect to gender, the different proportion of male/female in HFrEF and HFpEF was statistically significant with $p$ $<0.01$. No significant difference in smoking and alcohol use was found between any two of these three HF groups.

In terms of vital signs, pulse was significantly lower in patients with HFpEF than patients with $\operatorname{HFrEF}(p<0.01)$ while systolic blood pressure (SBP) was significantly higher in patients with HFpEF than patients with HFrEF $(p<0.01)$. SBP in patients with HFmrEF was significantly higher than patients with HFrEF $(p<0.05)$. The only difference for body mass index (BMI) in these $3 \mathrm{HF}$ groups was found between HFpEF and HFmrEF groups, whereas diastolic blood pressure (DBP) and body temperature were not significantly different among all the HF groups.

Table 1. Baseline characteristics and vital signs among the 3 groups 


\begin{tabular}{|c|c|c|c|c|c|c|c|}
\hline & All Patients & $\begin{array}{l}\text { HFrEF } \\
\text { (A. } n=93)\end{array}$ & $\begin{array}{l}\text { HFmrEF } \\
\text { (B. } n=56)\end{array}$ & $\begin{array}{l}\text { HFpEF } \\
\text { (C. } n=1081 \text { ) }\end{array}$ & $\begin{array}{c}P(A v \\
B)\end{array}$ & $\begin{array}{l}P(A v \\
C)\end{array}$ & $\begin{array}{l}P(B \mathrm{v} \\
\mathrm{C})\end{array}$ \\
\hline & 60.13 & & & 63.26 & & & \\
\hline LVEF (\%) & (25.13) & $33.27(4.81)$ & $44.16(2.58)$ & $(25.12)$ & $<.01$ & $<.01$ & $<.01$ \\
\hline Male & $826(67.15)$ & $77(82.80)$ & $43(76.79)$ & $706(65.31)$ & NS & $<.01$ & NS \\
\hline Female & $404(32.85)$ & $16(17.20)$ & $13(23.21)$ & $375(34.60)$ & NS & $<.01$ & NS \\
\hline Smoke & $309(25.12)$ & $32(34.41 \square$ & $19(33.93)$ & $258(23.87)$ & NS & NS & NS \\
\hline Alcohol & $195(15.85)$ & $20(21.51)$ & $10(17.86)$ & $165(15.26)$ & NS & NS & NS \\
\hline Age & $78.28(9.00)$ & $75.41(9.23)$ & $77.05(9.61)$ & $78.60(8.91)$ & NS & $<.05$ & NS \\
\hline $\operatorname{BMI}\left(\mathrm{kg} / \mathrm{m}^{2}\right)$ & $\begin{array}{l}24.84 \\
(14.29)\end{array}$ & $23.80(5.36)$ & $26.08(2.93)$ & $\begin{array}{l}24.87 \\
(15.27)\end{array}$ & NS & NS & $<.05$ \\
\hline Pulse(bpm) & $\begin{array}{l}76.16 \\
(14.66)\end{array}$ & $\begin{array}{l}84.09 \\
(19.59)\end{array}$ & $\begin{array}{l}80.08 \\
(17.64)\end{array}$ & $\begin{array}{l}75.32 \\
(13.79)\end{array}$ & NS & $<.01$ & NS \\
\hline $\mathrm{SBP}(\mathrm{mmHg})$ & $\begin{array}{l}129.74 \\
(29.31)\end{array}$ & $\begin{array}{l}119.95 \\
(20.45)\end{array}$ & $\begin{array}{l}135.34 \\
(23.74)\end{array}$ & $\begin{array}{l}130.29 \\
(30.01)\end{array}$ & $<.01$ & $<.01$ & NS \\
\hline $\mathrm{DBP}(\mathrm{mmHg})$ & $\begin{array}{l}72.79 \\
(12.06)\end{array}$ & $\begin{array}{l}71.68 \\
(12.14)\end{array}$ & $\begin{array}{l}73.98 \\
(12.02)\end{array}$ & $\begin{array}{l}72.82 \\
(12.06)\end{array}$ & NS & NS & NS \\
\hline $\begin{array}{l}\text { Temperature } \\
\left({ }^{\circ} \mathrm{C}\right)\end{array}$ & $36.44(0.33)$ & $36.43(0.26)$ & $36.47(0.40)$ & $36.44(0.33)$ & NS & NS & NS \\
\hline
\end{tabular}

Categorical variables were presented as Count (\%). Continuous variables were presented as Mean (SD).SBP: Systolic blood pressure; DBP: Diastolic blood pressure; BMI: Body mass index; bpm: Beat per minute; NS: Not significant.

\subsection{Comorbidity}

Comorbid diseases and interventions included but not limited to malignancy, hypertension, chronic obstructive pulmonary disease (COPD), diabetes mellitus, dyslipidemia, atrial fibrillation, anemia, percutaneous coronary intervention ( $\mathrm{PCl})$, pacemaker and cerebrovascular accident/transient ischemic attack(CVA/TIA), unstable angina pectoris (UAP), high uric acid and gout (HUGA). The frequency of all the comorbidities above had no significant difference between patients with HFmrEF and HFrEF. 
However, patients with HFpEF more frequently had comorbid peripheral artery disease $\mathrm{PAD})(\mathrm{p}<0.05)$, but less frequently had acute myocardial infarction(AMI) $(p<0.05)$, comorbid kidney disease (CKD) $(p<0.05)$, coronary artery disease $(C A D)(p<0.01)$ than those with HFrEF. Patients with HFmrEF more frequently had comorbid AMI $(p<0.01)$, infection $(p<0.05)$ and CAD $(p<0.05)$ than those with HFpEF. The result of pairwise comparisons of comorbid diseases among the three HF groups werelisted in Table 2.

Table 2. Comorbidities among the 3 groups 


\begin{tabular}{|c|c|c|c|c|c|c|c|}
\hline Comorbidity & All Patients & $\begin{array}{c}\text { HFrEF } \\
\text { (A. } n=93)\end{array}$ & $\begin{array}{c}\text { HFmrE } \\
\text { (B. } \mathrm{n}=56)\end{array}$ & $\begin{array}{c}\text { HFpEF } \\
\text { (C. } \\
\mathrm{n}=1081 \text { ) }\end{array}$ & $\begin{array}{c}P(A v v \\
B)\end{array}$ & $\begin{array}{c}P(A v \\
C)\end{array}$ & $\begin{array}{c}P(B \mathrm{v} \\
\text { C) }\end{array}$ \\
\hline Malignancy & $136(11.06)$ & $12(12.90)$ & $5(8.93)$ & $119(11.01)$ & NS & NS & NS \\
\hline Hypertension & $815(66.26)$ & $52(55.91)$ & $36(64.29)$ & $727(67.25)$ & NS & NS & NS \\
\hline COPD & $54(4.39)$ & $2(2.15)$ & $1(1.79)$ & $51(4.72)$ & NS & NS & NS \\
\hline Dyslipidemia & $201(16.34)$ & $8(8.60)$ & $7(12.50)$ & $186(17.21)$ & NS & NS & NS \\
\hline Atrial & $233(18.94)$ & $20(21.51)$ & $16(28.57)$ & $197(18.22)$ & NS & NS & NS \\
\hline \multicolumn{8}{|l|}{ fibrillation } \\
\hline Diabetes & $426(34.63)$ & $36(38.71)$ & $20(35.71)$ & $370(34.23)$ & NS & NS & NS \\
\hline \multicolumn{8}{|l|}{ mellitus } \\
\hline CKD & $180(14.63)$ & $23(24.73)$ & $13(23.21)$ & $144(13.32)$ & NS & $<.05$ & NS \\
\hline Anemia & $96(7.81)$ & $11(11.83)$ & $3(5.36)$ & $82(7.59)$ & NS & NS & NS \\
\hline AMI & $88(7.15)$ & $16(17.20)$ & $11(19.64)$ & $61(5.64)$ & NS & $<.05$ & $<.01$ \\
\hline Infection & 208(16.91) & $23(24.73)$ & $17(30.36)$ & $168(15.54)$ & NS & NS & $<.05$ \\
\hline PCI & $88(7.45) *$ & $12(12.90)$ & $6(10.71)$ & $70(6.48)$ & NS & NS & NS \\
\hline Pacemaker & $70(5.69) *$ & $4(4.30)$ & $6(10.71)$ & $60(5.55)$ & NS & NS & NS \\
\hline \multirow[t]{2}{*}{ CVA_TIA } & $244(20.64)$ & $16(17.20)$ & $12(4.55)$ & $216(19.98)$ & NS & NS & NS \\
\hline & * & & & & & & \\
\hline PAD & $428(34.80)$ & $21(22.58)$ & $16(28.57)$ & $391(36.17)$ & NS & $<.05$ & NS \\
\hline CAD & $723(58.78)$ & $70(75.27)$ & $42(75.00)$ & $611(56.52)$ & NS & $<.01$ & $<.05$ \\
\hline UAP & $73(5.94)$ & $6(6.45)$ & $2(3.57)$ & $65(6.01)$ & NS & NS & NS \\
\hline HUAG & $154(12.52)$ & 18(19.35) & $8(14.29)$ & $128(11.84)$ & NS & NS & NS \\
\hline
\end{tabular}

Data was presented as Count (\%).

*: Only 1182 patients had information on PCI, Pacemaker and CVA-TIA. 
Laboratory assessments were performed for all enrolled HF patients and the pair-wise comparisons among the 3 groups was listed in Table 3. The levels of NT-proBNP, and BNP were the value of log transformation before analysis. The following laboratory parameters:NT-proBNP,BNP,cardiac troponin,WBC, neutrophil\%, BUN, serum sodium, uric acid,weresignificantly higher in patients with HFmrEF than those with HFpEF.In addition, NT-proBNP, LDH, BNP,WBC, neutrophil\%,BUN, serum creatinine, serum sodium, D-dimer were also significantly higher in patients with HFrEF than those with HFpEF.But the levels ofcreatinekinase-MB and cardiac troponin were significantly higher in patients with HFpEF than with HFrEF.As for uric acid, there was no significant difference between these two groups. Meanwhile, the levels of BNP, LDH, and serum creatinine were significantly higher in patients with HFrEF than those of HFmrEF.

The other lab parameters observed but not mentioned above, including C-reactive protein, fasting blood glucose (FBP),serum potassium, hemoglobin etc., were comparable between any two of the three HF groups without any significant difference.

Table 3. Laboratory Parameters among the 3 groups 


\begin{tabular}{|c|c|c|c|c|c|c|c|}
\hline Lab Variable & All Patients & $\begin{array}{l}\text { HFrEF } \\
\text { (A. } n=93)\end{array}$ & $\begin{array}{l}\text { HFmrEF } \\
\text { (B. } n=56)\end{array}$ & $\begin{array}{l}\text { HFpEF } \\
\text { (C. } \\
n=1081 \text { ) }\end{array}$ & $\begin{array}{c}P(A v ~ \\
B)\end{array}$ & $\begin{array}{c}P(A v \\
C)\end{array}$ & $\begin{array}{c}P(B \mathrm{v} \\
\mathrm{C})\end{array}$ \\
\hline Log (NT-proBNP) & $6.21(1.37)$ & $9.81(0.52)$ & 7.49 (1.35) & $6.00(1.16)$ & NS & $<.05$ & $<.05$ \\
\hline $\log (\mathrm{BNP})$ & $4.77(1.05)$ & $6.68(0.95)$ & $5.95(1.16)$ & $4.57(0.85)$ & $<.01$ & $<.01$ & $<.01$ \\
\hline CK-MB (ng/mL) & $21.0(74.0)$ & $7.0(28.5)$ & $23.8(68.1)$ & $22.1(77.2)$ & NS & $<.05$ & NS \\
\hline $\mathrm{LDH}(\mathrm{U} / \mathrm{L})$ & $\begin{array}{l}212.4 \\
(188.8)\end{array}$ & $\begin{array}{l}326.2 \\
(271.7)\end{array}$ & $\begin{array}{l}256.2 \\
(208.1)\end{array}$ & $\begin{array}{l}202.3 \\
(177.7)\end{array}$ & $<.05$ & $<.01$ & NS \\
\hline
\end{tabular}

$\begin{array}{lllllllll}\text { Cardiac } & \text { troponin } 2.0(18.2) & 1.0(3.8) & 11.9(40.4) & 1.6(17.4) & \text { NS } & <.01<.01\end{array}$ $(\mathrm{ng} / \mathrm{mL})$

$\operatorname{WBC}\left(10^{9} / \mathrm{L}\right) \quad 6.0(3.2) \quad 6.9(3.7) \quad 7.3(4.1) \quad 5.8(3.0) \quad$ NS $\quad<.05 \quad<.05$

Neutrophil (\%) $\quad 60.6(12.8) 66.1(13.3) 67.2(14.5) 59.9(12.5) \quad$ NS $\quad<.01 \quad<.01$

$\begin{array}{llllll}\text { C-reactive } & \text { protein } 19.8(48.1) & 38.9(89.6) & 69.1 & 16.7(35.9) & \text { NS }\end{array}$ NS $\quad$ NS $(\mathrm{mg} / \mathrm{L})$

(133.3)

$\begin{array}{llllllll}\mathrm{BUN}(\mathrm{mmol} / \mathrm{L}) & 7.6(4.2) & 10.3(5.7) & 8.4(4.1) & 7.4(4.0) & \mathrm{NS} & <.01 & <.01\end{array}$

$\begin{array}{lllllllllll}\text { Serum } & \text { creatinine } & 78.1(72.9) & 118.5 & 83.0(53.7) & 74.8(68.5) & <.05 & <.01 & \text { NS }\end{array}$

(umol/L) (113.9)

$\begin{array}{llllllll}\text { Serum } & \text { Sodium } 138.8 & 139.2(4.2) & 139.8(3.7) & 138.7 & \text { NS } & <.01 & <.05\end{array}$

$(\mathrm{mmol} / \mathrm{L}) \quad$ (15.5) (16.5)

$\begin{array}{lllllll}\text { Serum Potassium } & 5.3(3.1) & 4.8(2.4) & 4.8(2.9) & 5.3(3.2) & \text { NS } & \text { NS }\end{array} \quad$ NS

$(\mathrm{mmol} / \mathrm{L})$

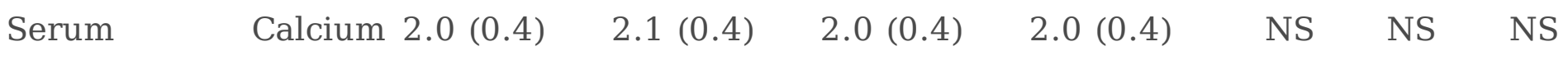

$(\mathrm{mmol} / \mathrm{L})$

FBP (mmol/L) $\quad 8.1(3.0) \quad 6.7(1.5) \quad 11.1(5.2) \quad 8.1(2.9) \quad$ NS $\quad$ NS $\quad$ NS

Hemoglobin A1c (\%) $\quad 6.8(4.7) \quad 6.4(1.0) \quad 6.6(1.6) \quad 6.9(4,8) \quad$ NS $\quad$ NS $\quad$ NS

$\begin{array}{llllllll}\text { Hemoglobin }(\mathrm{g} / \mathrm{L}) & 135.3 & 133.2 & 128.2 & 135.8 & \text { NS } & \text { NS } & \text { NS } \\ & (37.3) & (34.4) & (30.5) & (37.8) & & & \end{array}$




\begin{tabular}{llllllll} 
D-dimer (ug/ml) & $1.3(2.1)$ & $2.1(3.1)$ & $1.2(1.4)$ & $1.3(2.0)$ & NS & $<.01$ & NS \\
TSH $(\mathrm{mIU} / \mathrm{L})$ & $2.3(3.4)$ & $2.5(1.8)$ & $2.3(3.5)$ & $2.3(3.4)$ & NS & NS & NS \\
& & & & & & & \\
Uric acid (umol/L) & 279.6 & 339.1 & 330.4 & 274.9 & NS & NS & $<.05$ \\
& $(165.0)$ & $(228.4)$ & $(160.4)$ & $(161.2)$ & & & \\
\hline
\end{tabular}

Data was presented as Mean (SD). Independent t-test was used to compare the lab variables.

Log (NT-proBNP): Log transformation of NT-proBNP; Log (BNP): Log transformation of BNP; CK-MB: Creatine kinaseMB; LDH: Lactic acid dehydrogenase;WBC: White blood cell; BUN, Blood urea nitrogen; FBP: Fasting blood glucose; TSH:Thyrotropin stimulating hormone; NS: Not significant

\subsection{Mortality rate}

The mortality result of HFmrEF, HFpEF and HFrEF patients are shown in Table 4. There were 92 overall deaths $(7.47 \%)$ in the HF patients during the study period, with a slightly higher unadjusted mortality rate seen in HFmrEF group (10.71\%) than in HFpEF group (7.31\%) or in HFrEF group (7.53\%). However, there was no significant difference for the mortality rate among these three HF groups.

Table 4. Mortality rate among the 3 groups

\begin{tabular}{|c|c|c|c|c|c|c|c|}
\hline & All patients & HFrEF & HFmrEF & HFpEF & \multirow{2}{*}{$P(A \vee B$} & $(\mathrm{Av}$ & $(B \mathrm{v} C)$ \\
\hline & \multicolumn{4}{|c|}{ 1. $\mathrm{n}=93) 1 \cdot \mathrm{n}=56)$ 1. $\mathrm{n}=1081)$} & & & \\
\hline Death & $92(7.47)$ & $7(7.53)$ & $6(10.71)$ & $79(7.31)$ & NS & NS & NS \\
\hline
\end{tabular}

Data was presented as count (\%). The $\chi^{2}$ test was used to compare variables.

\subsection{Risk factors associated with HFmrEF,HFrEF and HFpEF}

The univariate analysis of the associated factors with HFmrEF, HFpEF and HFrEF was shown asforest plots inFigure 2 and multivariable logistic regression analysis was performed to obtainthe odds ratios(Table 5).

Table 5. Mortality of 3 groups adjusted for risk factors 


\begin{tabular}{|c|c|c|c|c|c|c|c|c|}
\hline \multirow[b]{2}{*}{ Type } & \multicolumn{2}{|c|}{ Model 1} & \multicolumn{2}{|c|}{ Model 2} & \multicolumn{2}{|c|}{ Model 3} & \multicolumn{2}{|c|}{ Model 4} \\
\hline & OR $(95 \% C I)$ & $\mathbf{P}$ & OR (95\%CI) & $\mathbf{P}$ & OR (95\%CI) & $\mathbf{P}$ & $\begin{array}{c}\text { OR } \\
(95 \% \mathrm{CI})\end{array}$ & $\mathbf{P}$ \\
\hline HFpEF & Referen & & Reference & & Reference & & Reference & \\
\hline HFmrEF & $\begin{array}{c}1.55(0.58 \\
3.51)\end{array}$ & 0.33 & $\begin{array}{l}1.52(0.57 \\
3.46)\end{array}$ & 0.35 & $\begin{array}{l}1.32(0.48 \\
3.03)\end{array}$ & 0.097 & $\begin{array}{l}1.31(0.48, \\
3.02)\end{array}$ & 0.35 \\
\hline HFrEF & $\begin{array}{c}1.11(0.45 \\
2.36)\end{array}$ & 0.80 & $\begin{array}{l}1.11(0.45, \\
2.36)\end{array}$ & 0.80 & $\begin{array}{l}0.97(0.39, \\
2.08)\end{array}$ & 0.161 & $\begin{array}{l}0.94(0.37, \\
2.03)\end{array}$ & 0.80 \\
\hline
\end{tabular}

OR: oddsratio; CI: confidence interval.

Model 1: adjusted for age and gender. Model 2: adjusted for age, gender, smoke and alcohol. Model 3: adjusted for age, gender, smoke, alcohol and baseline cardiovascular disease. Model 4: adjusted for age, gender, smoke, alcohol and baseline cardiovascular disease, COPD, diabetes and hypertension.

The resultsof univariate and multivariable logistic regression indicatedthatcomorbidatrial fibrillation, AMI and infectionwere associated with HFmrEF, whereas female gender, old age, comorbid hypertension and PAD were associated with HFpEF, and CAD, kidney disease were associated with HFrEF (see Table 6).

Among all baseline characteristics and clinical parameters, AMI had the highest odds ratio for HFmrEF (4.21), while female and CAD had the highest odds ratio for HFpEF (2.55) and HFrEF (3.02), respectively.

Our univariate analysis also indicated that baseline characteristics, comorbid features and laboratory results were comparable between HFmrEF patients and HFrEF patients, which were displayed in Supplemental Figure 1 after the reference section.

Table 6. Risk factors associated with HFmrEF, HFpEF and HFrEF. 


\begin{tabular}{lcc}
\hline For HFmrEF & OR $(95 \%$ CI $)$ & $P$ \\
\hline Atrial fibrillation & $1.86(1.01,3.42)$ & 0.05 \\
AMI & $4.21(2.06,8.63)<.01$ \\
Infection & $2.37(1.30,4.32)<.01$ \\
\hline For HFpEF & \\
\hline Sex (Female $)$ & $2.55(1.45,4.50)<.01$ \\
Age & $1.05(1.02,1.07)<.01$ \\
Hypertension & $2.36(1.46,3.82)<.01$ \\
PAD & $1.89(1.11,3.22)$ & 0.02 \\
\hline For HFrEF & \\
\hline CAD & $3.02(1.80,5.08)<.01$ \\
Kidney disease & $2.19(1.25,3.84)<.01$ \\
\hline
\end{tabular}

\section{Discussion}

Our study was a single-center, retrospective study conducted in Shenyang, a provincial capital city in northeastern China,to provide data on the demographics and clinical characteristics of geriatrics inpatients with HFrEF, HFmrEF and HFpEF.HFmrEF, as a newly defined HF type by ESC in2016, was investigated and compared with other HF types, revealing that patients with HFmrEF was closer to patients with HFrEF than patients with HFpEF in terms of most of the clinical baseline characteristics.

In our study, HFpEF accounted for $87.8 \%$ of all HF patients included, while HFmrEF and HFrEF accounted for $4.5 \%$ and $7.5 \%$ respectively. The prevalence of HFmrEF $₫ .5 \% \bigotimes$ in our study was muchlower thanin other studies $(16 \%, 24 \%$ or $17 \%$ [ [7-9].Previous studies were mostly based on the statistical results of patients with $\mathrm{HF}$, or registered in hypertension, HF centers and other related data[10-12]. In contrast, this study was on inpatients of geriatrics department, so it is much more asymptotic to the incidence of $\mathrm{HF}$ in the elderly population, and will be more conducive to reminding geriatricians of identifying HF patients, and following up analysis on HFpEF patients with a large proportion, and exploring more factors affecting heart function to further guide the clinical treatment of HFpEF[5].The mean age of HF patients included was 78.28, higher than those reported in other studies[5],which could be explained by the fact 
that our patients were enrolled from the geriatric department of a hospital where usually older patients are admitted.Previous studies including older patients showed a higher frequency of HFpEF[13]. And our finding was in accordance with that. ThatHFpEFpatients were older than HFmrEF and HFrEF patients (78.6 vs 77.05 and 75.41).In addition,our patients were certainly from geriatrics department, not just cardiovascular department.This may be the reason for the largest proportion of HFpEF.Asub-cohort of the PROTECT trial the proportion of patients with HFpEF is $13 \%$. The included patients have a history of HF, who were admitted with acute $\mathrm{HF}$ and mild to moderate renal dysfunction,age $\geq 18$ years.[14].In aretrospective study from Japan,HFrEFロHFmrEF and HFpEF accounted for $36 \%, 21 \%$ and $43 \%$ respectively.Patients included in this study were all admittedfor acute decompensated HF[15].

In Turkey-the APOLLON study, patients with HFpEF were more likely to be women, withlower frequency of coronary artery disease and myocardial infarction and kidney disease than patients with HFrEF[16]. Similarly, in our study, women accounted for a higher frequency of HFpEF, which is consistent with this, considering that this phenomenon may be related to the greater exposure of male patients to cardiovascular disease risk factors[17].

The acute myocardial infarction and kidney disease including both chronic kidney disease and acute kidney injury were analyzed in our study. Our study showed that atrial fibrillation was most frequently observed in patients with HFmrEF (28.57\%), while it was mostly observed in patients with HFpEF (38.2\%) in the APOLLON study[16]. The frequency of atrial fibrillation in the present study appeared lower than another study from Swedish Heart Failure Registry(21.51\%vs 53\%, $28.57 \%$ vs $60 \%$ and 18.22 vs $65 \%$ ) in HFrEF, HFmrEF and HFpEF patients respectively[18], but slightly higher than the CHARM study (Candesartan in Heart failure-Assessment of Reduction in Mortality and morbidity) with the prevalence of AF $19 \%$ in HFpEF and $17 \%$ in HFrEF[19]. One reason for the different prevalence of AF among these studies might be the different cohort selection. Our patients are all from the geriatrics department, who are older and have a higher incidence of atrial fibrillation.

When comparing the three HF patient groups for comorbidities, we either found that they were differently distributed between patients with HFmrEF and HFpEF (AMI, infection, CAD), or between HFpEF and HFrEF (kidney disease, AMI, PAD and CAD), but failed to find any significant difference betweenpatients with HFmrEF and HFrEF for all these comorbidities. Moreover, our multivariable analysis showed that CAD was associated with HFrEF, while AMI, as a part of CAD, was associated with HFmrEF. A few previous studies also showed that the frequency of CAD in HFmrEF patients was similar toHFrEF patients, both higher than HFpEF patients[20-22].

Similar to the APPLON study, some comorbidities including malignancy, COPD (chronic obstructive pulmonary disease), diabetes mellitus, anemia and CVA_TIA (cere brovascular accident/transient ischemic attack) were comparable between HFmrEF patients and HFpEF patients [16].Previous studies have shown hypertension was more frequently observed in HFpEF patients. But in our study, hypertension showed a similar tendency with a higher percentage found in HFpEF patients than HFrEF and HFmrEF 
patients, even not statistically significantly. In our multivariable analysis, indeed hypertension was found to be associated with HFpEF besides female, old age and PAD (peripheral artery disease).

The level of NT-proBNP, BNP,blood urea nitrogen, creatinine was significantly lower in patients with HFpEF than those with HFmrEF or HFrEF, which was in accordance with the previous studies $[16,23]$. This may be explained with the prevalence of kidney disease tending to be higher in HFmrEF or HFrEF patients than in HFpEF patients.

However, some studies have presented different results. Josephine et al. performed a meta-analysis on the characteristics and prognosis of HFrEF, HFmrEF and HFpEF patients and concluded that HFmrEF was a distinctive category in between HFrEF and HFpEF[24].In a prospective study based on the Sweden HF Registry cohort, HFmrEF resembled HFrEF for some characteristics, but resembled HFpEF for some other characteristics and HFmrEF was concluded to be an intermediate phenotype between HFrEF and HFpEF[21].In our study, HFmrEF showed no significant difference with HFrEF in terms of all the comorbidities observed and most of the lab parameters measured including NT-proBNP, creatine kinase$M B$, cardiac troponin, uric acid, WBC etc. Our study revealed that a similar overall disease profile might be shared in between patients with HFmrEF and HFrEF. HFmrEF was a disease entity more similar toHFrEF than to HFpEF, which seemed to be contradictory to these previous studies[24,21]. But considering the difference in the proportion of HFrEF, HFmrEF and HFpEF $(56 \%, 21 \%$ and $23 \%$ in the Sweden HF study and $7.5 \%, 4.5 \%$ and $87.8 \%$ in our study) and the difference in the overall HF population selection (from a HF registry covering all Sweden and from geriatric department of a hospital in China) in the two studies, and other underlying differences in the operation and process of the related data, it is not surprising that the results may differ somehow. At the very least, our study provided a new evidence about the distribution of related variables among different HF types in this particular HF population, and may potentially contribute to the treatment decision toward HF in the future.

Our finding suggested that the mortality rate was comparable among all three HF groups, in consistent with previous studies where comparable mortality was observed in HFpEF and HFrEFgroups [25]. But the overall mortality in current study was lower $(7.22 \%)$ for patients with HFpEF than in other studies showing a one-year mortality of 19 to $29 \%$ [26]. One explanation for this was that our mortality rate was based on the in-hospital deaths only, while theirs was based on both in and out of the hospital with follow-up data collected[26]. Notably our study lacked etiology mortality analysis, so what caused these deaths among these HF patients remained unclear. With the complexity and heterogeneity of HF, apparently more comprehensive studies are needed to better determine the long-term prognosis of patients according to etiology.

\section{Limitation}

The results of the present study should be interpreted in the context of certain limitations.First, it was a retrospective study, lacking of follow-upinformation and the patient's out-of-hospital death record, so all the data were analyzed on the basis of hospitalization period only. We assessed associations between 
clinical characteristics and LVEFgroups, but we could not demonstrate causality and survival model for the prognosis study.Our multivariable analysis showed thatatrial fibrillation, AMI and infectionwere the risk factors associated with HFmrEF, while female, old age, hypertension and PAD were associated with HFpEF, and CAD and kidney disease were associated with HFrEF, but we could not conclude whether these conditions or factors preceded HF or were consequences of HF.

Secondly, the number of patients with HFmrEF and HFrEF was relatively small so thatthe interpretation of our results might be limited. Therefore, future trials will require increasing enrollment of patients with HFrEF and HFmrEFto better understand the characteristics and clinical outcomes and eventually help evaluate possible therapeutic options for HF patients.

Thirdly, we did not perform etiology analysis for HF. If this could be included in the future studies, it might be helpful for the pathophysiology study of HFpEF and HFmrEF, thus contributing to the medication selection.

Finally, the study participants were from a single hospital in China, despite of its very first rank in general hospitals in northeastern China, it is still uncertain whether these findings can be generalized to patients in other areas of China.

\section{Conclusions}

This was a retrospective study conducted on HF patients in a hospital located in northeastern China. Heart failure with preserved ejection fraction(HFpEF, LVEF>50\%)represented the majority of all cases of $\mathrm{HF}$ in the present study. Heart failure with mid-range ejection fraction (HFmrEF, LVEF40-49\%), as a newly defined type of HF, was closer to heart failure with reduced ejection fraction (HFrEF, LVEF $<40 \%$ ) in terms of most of the demographics, comorbidities and laboratory parameters observed, with certain differences found between HFmrEF and HFpEF groups, more so between HFrEF and HFpEF for these observed features. Overall, more comprehensive studies including higher proportion of patients with HFmrEF, follow-up study are needed to better understand this newly defined HF category, to further identify the determinants accounting for its significant potential differences from the other two HF groups, survival analysis and ultimately to guide beneficial therapies.

\section{Abbreviations}

HF: Heart failure; HFmrEF: HF with mid-range ejection fraction; HFpEF: HF with preserved ejection fraction; HFrEF: HF with reduced ejection fraction; ESC: European Society of Cardiology; NT-proBNP: Nterminal pro B-type natriuretic peptide; BNP: brain natriuretic peptide; BUN: blood urea nitrogen; WBC: white blood cell; LDH: lactic acid dehydrogenase; LDL: low-density lipoprotein; HDL: high-density lipoprotein; LVEF: left ventricular ejection fraction; BMI: body mass index; CAD: Coronary artery disease; UAP: unstable angina pectoris; CKD: comorbid kidney disease; AMI: acute myocardial infarction; SBP: systolic blood pressure; DBP: diastolic blood pressure; COPD: chronic obstructive pulmonary disease; PCl: 
percutaneous coronary intervention; CVA: cerebrovascular accident ;TIA: transient ischemic attack; HUGA: high uric acid and gout; PAD: peripheral artery disease; FBP: fasting blood glucose; TSH: Thyrotropin stimulating hormone.

\section{Declarations}

\section{Ethics approval and consent to participate}

The study was approved by the ethics committee of the First Affiliated Hospital of China Medical University(\#2020232). All methods were performed in accordance with the relevant guidelines and regulations. The need for informed consent was waived because of the retrospective nature of the analysis.

\section{Consent for publication}

Not applicable.

\section{Availability of data and materials}

The datasets generated and analysed during the current study are not publicly available due fact that our project need to be continued, but are available from the corresponding author on reasonable request.

\section{Competing interests}

The authors declare that they have no competing interests.

\section{Funding}

There was no funding information available for this study.

\section{Authors' contributions}

$\mathrm{CZH}$ and ZWZ made main contribution to design of the study, drafted the manuscript,and performed the statistical analysis and participated. XOW participated in the data collection. WT revised the manuscript, and gave critical comments on the draft.All authors have read and approved the final manuscript.

\section{Acknowledgements}

None.

\section{Author details}

1 Department of Geriatrics, the first affiliated hospital of China medical university, Shenyang, China.

\section{References}


1. Yancy CW, Jessup M, Bozkurt B, Butler J, Casey Jr. DE, Drazner MH, et al. 2013 ACCF/AHA guideline for the management of heart failure: executive summary: a report of the American College of Cardiology Foundation/American Heart Association Task Force on practice guidelines. Circulation 2013 Oct 15;128(16):1810-52.

2. Hunt SA, Abraham WT, Chin MH, Feldman AM, Francis GS, Ganiats TG, Jessup M, Konstam MA, Mancini DM, Michl K, Oates JA, Rahko PS, Silver MA, Stevenson LW, Yancy CW. 2009 Focused update incorporated into the ACC/AHA 2005 Guidelines for the Diagnosis and Management of Heart Failure in Adults: a report of the American College of Cardiology Foundation/American Heart Association Task Force on Practice Guidelines: developed in 1 collaboration with the International Society for Heart and Lung Transplantation. Circulation. 2009;119: e391-e479.

3. Ponikowski P, Voors AA, Anker SD, Bueno H, Cleland JGF, Coats AJS, et al. ESC guidelines for the diagnosis and treatment of acute and chronic heart failure: the Task Force for the diagnosis and treatment of acute and chronic heart failure of the European Society of Cardiology (ESC)developed with the special contribution of the Heart Failure OAssociation (HFA) of the ESC. Eur Heart $J$ 2016;37(27):2129-200.

4. Butler J, Fonarow GC, Zile MR, Lam CS, Roessig L, Schelbert EB, Shah SJ, Ahmed A, Bonow RO, Cleland JGF, Cody RJ, Chioncel O, Collins SP, Dunnmon P, Filippatos G, Lefkowitz MP, Marti CN, McMurray JJ, Misselwitz F, Nodari S, O'Connor C, Pfeffer MA, Pieske B, Pitt B, Rosano G, Sabbah HN, Senni M, Solomon SD, Stockbridge N, Teerlink JR, Georgiopoulou VV, Gheorghiade M. Developing therapies for heart failure with preserved ejection fraction: current state and future directions. JACC Heart Fail 2014;2:97-112.

5. Mosterd A, Hoes AW. Clinical epidemiology of heart failure. Heart 2007;93: 1137-1146.

6. Redfield MM, Jacobsen SJ, Burnett JC Jr, et al. Burden of systolic and diastolic ventricular dysfunction in the community: appreciating the scope of the heartfailure epidemic. JAMA 2003;289:194-202.

7. Gómez-Otero I, Ferrero-Gregori A, Varela Román A, et al. Mid-range ejection fraction does not permit risk stratification among patients hospitalized for heart failure. Rev EspCardiol 2017;70:338-

8. Chioncel O, Lainscak M, Seferovic PM, et al. Epidemiology and one-year outcomes in patients with chronic heart failure and preserved, mid-range and reduced ejection fraction: an analysis of the ESC Heart Failure Long-Term Registry. Eur J Heart Fail. 2017;19(12):1574-1585.

9. Rickenbacher P, Kaufmann BA, Maeder MT, et al. Heart failure with mid-range ejection fraction: a distinct clinical entity? Insights from the Trial of Intensified versus standard Medical therapy in Elderly patients with Congestive Heart Failure (TIME-CHF). Eur J Heart Fail. 2017;19(12):1586-1596.

10. Lund LH, Claggett B, Liu J, Lam CS, Jhund PS, Rosano GM, Swedberg K, Yusuf S, Granger CB, Pfeffer MA, McMurray JJV , \& Solomon SD. Heart failure with mid-range ejection fraction in CHARM: characteristics, outcomes and effect of candesartan across the entire ejection fraction spectrum. European journal of heart failure. 2018; 20.8: 1230-1239. 
11. Huusko J, Tuominen S, Studer R, Corda S, Proudfoot C, Lassenius M, \&Ukkonen H. Recurrent hospitalizations are associated with increased mortality across the ejection fraction range in heart failure. ESC heart failure. 2020; 7.5: 2406-2417.

12. Oka T, Tanaka H, Murasaki S, Yamauchi T, Katoh J, Hattori H, Kikuchi N, Watanabe E, Yamada Y, Haruki S, Kogure T, Suzuki T, Uetsuka Y, Hagiwara N \& The HIJ-HF II Investigators. Clinical characteristics of hospitalized heart failure patients with preserved, mid-range, and reduced ejection fractions in Japan. ESC heart failure. 2019; 6.3: 475-486.

13. Hogg K, Swedberg K, McMurray J. Heart failure with preserved left ventricular systolic function; epidemiology, clinical characteristics, and prognosis. J Am CollCardiol 2004;43:317-27.

14. Tromp J, Khan MAF, Mentz RJ, et al. Biomarker Profiles of Acute Heart Failure Patients With a MidRange Ejection Fraction. JACC Heart Fail. 2017;5(7):507-517.

15. Shiga T, Suzuki A, Haruta S, et al. Clinical characteristics of hospitalized heart failure patients with preserved, mid-range, and reduced ejection fractions in Japan. ESC Heart Fail. 2019;6(3):475-486.

16. Özlek B, Özlek E, Ağuş HZ, et al. Patients with HFpEF and HFmrEF have different clinical characteristics in Turkey: A multicenter observational study. Eur J Intern Med. 2019;61:88-95.

17. Weidner $\mathrm{G}$. Why do men get more heart disease than women? An international perspective. J Am Coll Health. 2000 May;48(6):291-4. doi: 10.1080/07448480009596270. PMID: 10863872.

18. Sartipy U, Dahlström U, Fu M, Lund LH. Atrial fibrillation in heart failure with preserved, mid-range, and reduced ejection fraction. JACC Heart Fail 2017 Aug;5(8):565-

19. Olsson LG, Swedberg K, Ducharme A, et al.Atrial fibrillation and risk of clinical events inchronic heart failure with and without left ventricularsystolic dysfunction: results from theCandesartan in Heart failure-Assessment ofReduction in Mortality and morbidity (CHARM)program. J Am CollCardiol 2006;47:1997-2004.

20. Kapoor JR, Kapoor R, Ju C, Heidenreich PA, Eapen ZJ, Hernandez AF, etal.Precipitating clinical factors, heart failure characterization, and outcomes in patientshospitalized with heart failure with reduced, borderline, and preservedejection fraction. JACC Heart Fail. 2016 Jun;4(6):464-

21. Koh AS, Tay WT, Teng THK, Vedin O, Benson L, Dahlstrom U, et al. A comprehensive population-based characterization of heart failure with mid-range ejection fraction. Eur J Heart Fail 2017 Dec;19(12):1624-

22. Webb J, Draper J, Fovargue L, et al. Is heart failure with mid range ejection fraction (HFmrEF) a distinct clinical entity or an overlap group?.Int J Cardiol Heart Vasc. 2018;21:1-6. Published 2018 Sep 6. doi:10.1016/j.ijcha.2018.06.001

23. Maisel AS, McCord J, Nowak RM, Hollander JE, Wu AH, Duc P, Omland T, Storrow AB, Krishnaswamy P, Abraham WT, Clopton P, Steg G, Aumont MC, Westheim A, Knudsen CW,Perez A, Kamin R, Kazanegra R, Herrmann HC, McCullough PA. Bedside B-Type natriuretic peptide in the emergency diagnosis of heart failure with reduced or preserved ejection fraction. Results from the Breathing Not Properly Multinational Study. J Am CollCardiol. 2003; 41:2010- 2017. [PubMed: 12798574] 
24. Lauritsen J, Gustafsson F, Abdulla J. Characteristics and long-term prognosis of patients with heart failure and mid-range ejection fraction compared with reducedand preserved ejection fraction: a systematic review and meta-analysis. ESC Heart Fail 2018 Apr 16.

25. Nativi-Nicolau J, Ryan JJ, Fang JC. Current therapeutic approach in heart failure with preserved ejection fraction. Heart Fail Clin 2014 Jul;10(3):525-

26. Komajda M, Lam CS. Heart failure with preserved ejection fraction: a clinical dilemma. Eur Heart J 2014 Apr;35(16):1022-

\section{Figures}

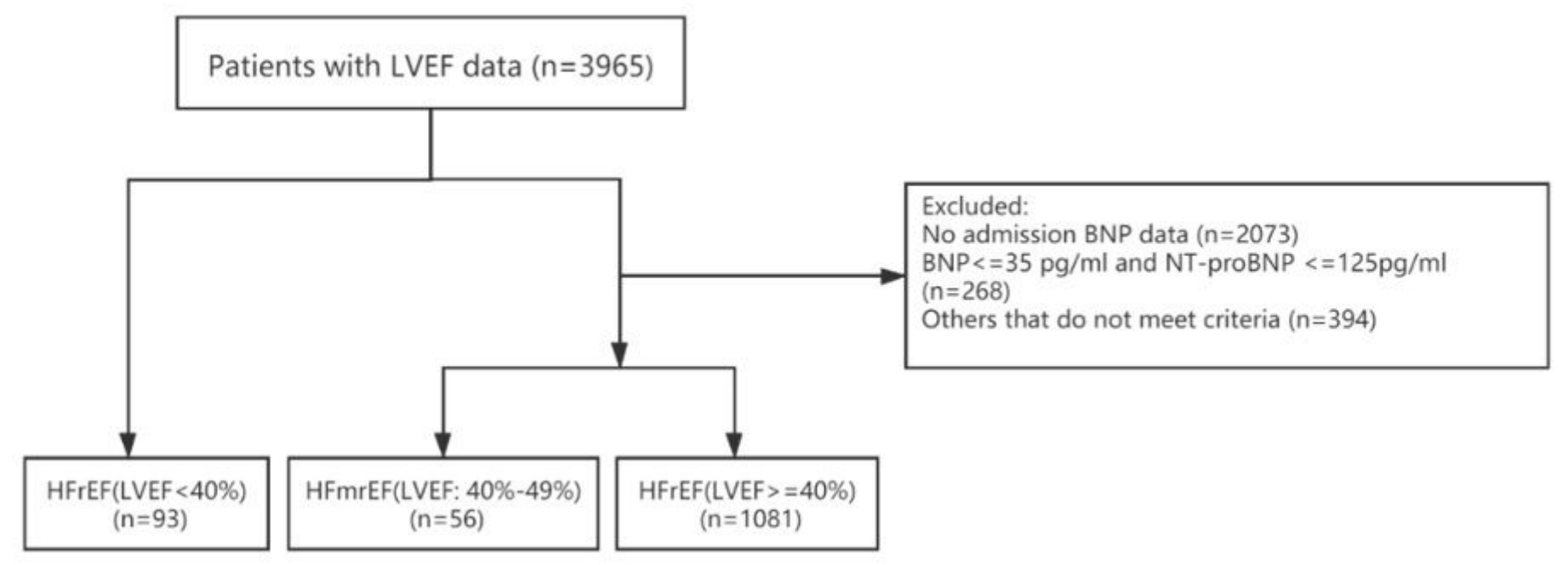

Figure 1

Flowchart of population selection. 
A: HFMrEF vs HFpEF

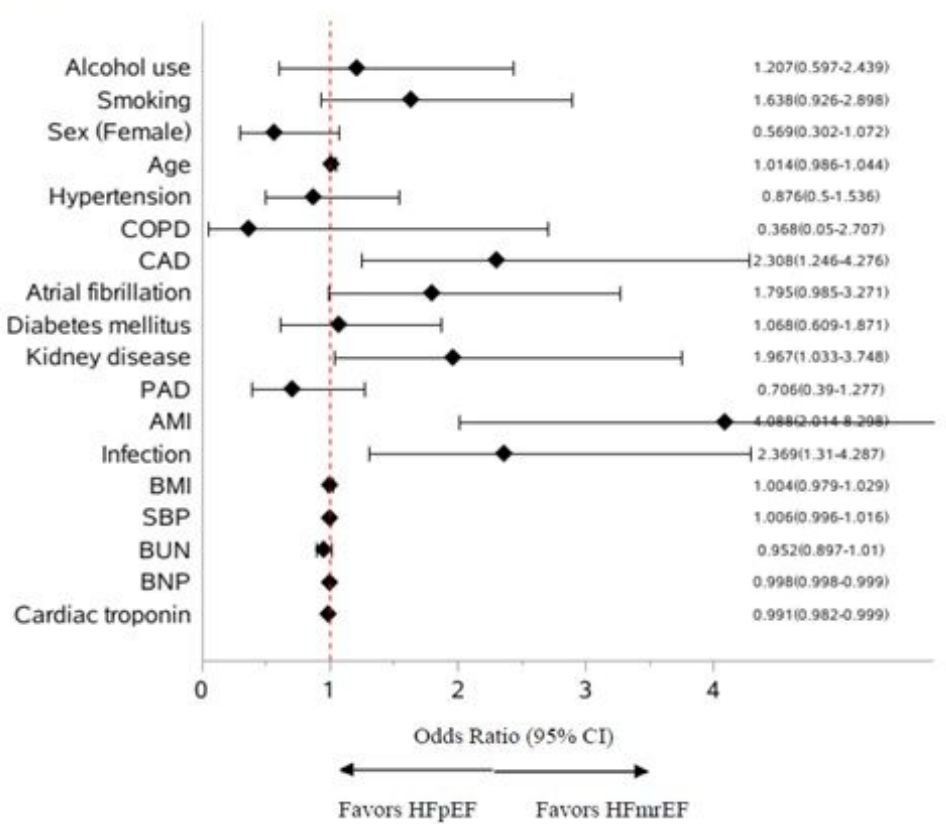

B: HFrEF vs HFpEF

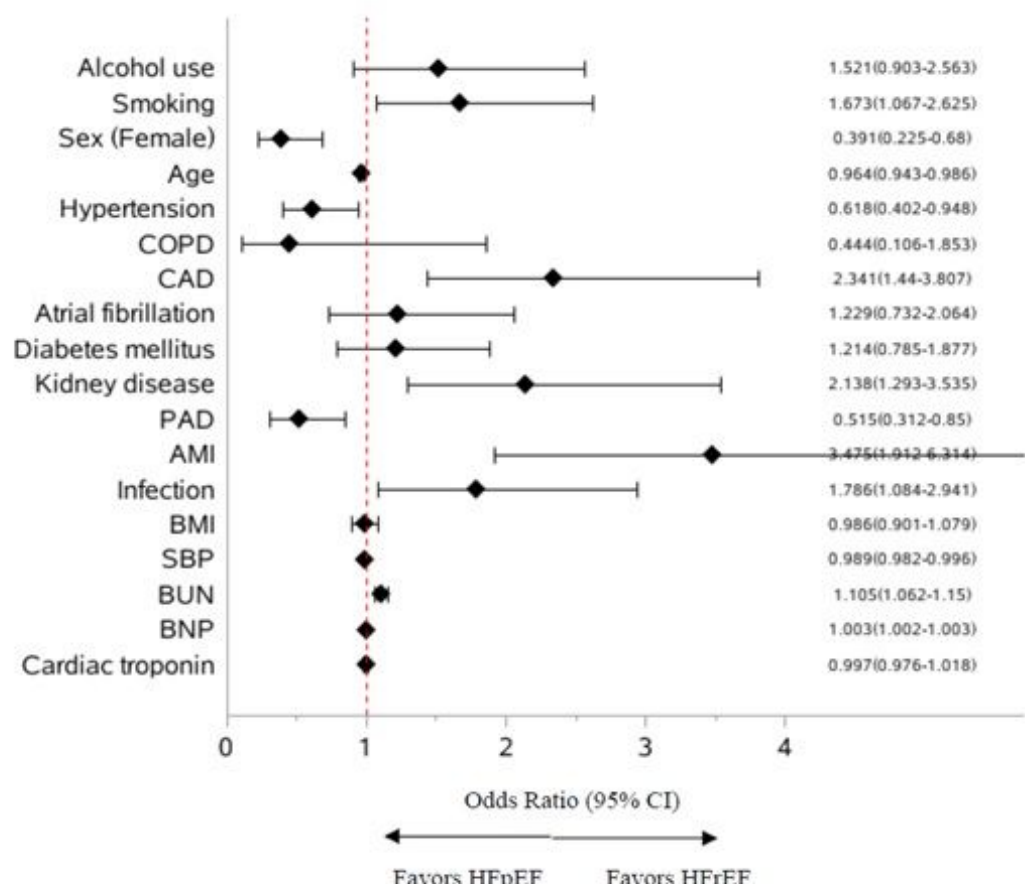

Figure 2

Forest plots of unadjusted odds ratios

\section{Supplementary Files}

This is a list of supplementary files associated with this preprint. Click to download. 
- Supplementarylnformation.doc

Page 21/21 